\title{
BMJ Open Cost-effectiveness of total knee replacement in addition to non-surgical treatment: a 2-year outcome from a randomised trial in secondary care in Denmark
}

To cite: Skou ST, Roos E, Laursen M, et al. Costeffectiveness of total knee replacement in addition to non-surgical treatment: a 2-year outcome from a randomised trial in secondary care in Denmark. BMJ Open 2020;10:e033495. doi:10.1136/ bmjopen-2019-033495

- Prepublication history and additional material for this paper are available online. To view these files, please visit the journal online (http://dx.doi. org/10.1136/bmjopen-2019033495).

Received 07 August 2019 Revised 14 November 2019 Accepted 31 December 2019

Check for updates

(C) Author(s) (or their employer(s)) 2020. Re-use permitted under CC BY-NC. No commercial re-use. See rights and permissions. Published by BMJ.

For numbered affiliations see end of article.

Correspondence to Dr Søren Thorgaard Skou; stskou@health.sdu.dk

\section{ABSTRACT}

Objective To assess the 24-month cost-effectiveness of total knee replacement (TKR) plus non-surgical treatment compared with non-surgical treatment with the option of later TKR if needed.

Methods 100 adults with moderate-to-severe knee osteoarthritis found eligible for TKR by an orthopaedic surgeon in secondary care were randomised to TKR plus 12 weeks of supervised non-surgical treatment (exercise, education, diet, insoles and pain medication) or to supervised non-surgical treatment alone. Including qualityadjusted life years (QALYs) data from baseline, 3, 6, 12 and 24 months, effectiveness was measured as change at 24 months. Healthcare costs and transfer payments were derived from national registries. Incremental healthcare costs, and incremental cost-effectiveness ratios (ICERs) were calculated. A probabilistic sensitivity analysis was conducted and the probability of cost-effectiveness was estimated at the 22665 Euros/QALY threshold defined by the National Institute for Health and Care Excellence. Results TKR plus non-surgical treatment was more expensive (mean of 23076 vs 14514 Euros) but also more effective than non-surgical treatment (mean 24-month improvement in QALY of 0.195 vs 0.056 ). While costeffective in the unadjusted scenario (ICER of 18497 Euros/QALY), TKR plus non-surgical treatment was not cost-effective compared with non-surgical treatment with the option of later TKR if needed in the adjusted (age, sex and baseline values), base-case scenario (ICER of 32611 Euros/QALY) with a probability of cost-effectiveness of $23.2 \%$. Including deaths, TKR plus non-surgical treatment was still not cost-effective (ICERs of 46277 to 64208 Euros/QALY).

Conclusions From a 24-month perspective, TKR plus non-surgical treatment does not appear to be costeffective compared with non-surgical treatment with the option of later TKR if needed in patients with moderateto-severe knee osteoarthritis and moderate intensity pain in secondary care in Denmark. Results were sensitive to changes, highlighting the need for further confirmatory research also assessing the long-term cost-effectiveness of TKR.
Strengths and limitations of this study

- This is the first economic evaluation of total knee replacement (TKR) that is based on a randomised trial of surgical and non-surgical treatment thereby providing highly comparable treatment groups assessed and treated in a standardised and controlled setup.

- Cost data were retrieved from the Danish health registries which contain detailed, high-quality information on health sector costs, social costs and prescription medication on individual patients, and effectiveness data were systematically and rigorously collected in the randomised trial.

- The 24-month time horizon and the selected population included limit conclusions on the long-term cost-effectiveness of total knee replacement and the generalisability to other populations.

- Since nearly one out of three from the non-surgical group had TKR surgery during the 24 months, it is likely that the true additional effect and cost of TKR in addition to non-surgical treatment have been underestimated in the study.

Trial registration number ClinicalTrials.gov (NCT01410409).

\section{INTRODUCTION}

Knee osteoarthritis (OA) is one of the leading contributors to the global burden of disease ${ }^{1}$ with considerable pain and functional limitations for the individual. ${ }^{2}$ The disease has been estimated to affect 250 million people worldwide, ${ }^{3}$ with total European costs estimated to be 817 billion Euros per year. ${ }^{4}$ Over the last 20 years, the prevalence of knee OA has increased substantially ${ }^{5}$ and is expected to continue to increase ${ }^{1}$ and amplify the societal burden. 
In patients with end-stage knee OA, total knee replacement (TKR) is considered an effective ${ }^{6}$ and cost-effective $^{7}$ treatment. However, approximately $20 \%$ continue to have chronic pain after otherwise successful surgery $^{8}$ and, in addition, the procedure is associated with a risk of serious adverse events. ${ }^{9}$ Furthermore, clinical guidelines reflecting high-quality evidence from recent decades highlight non-surgical treatments as an effective and less costly treatment for patients with knee OA. ${ }^{10}$ As the number of TKR procedures performed each year has increased dramatically since the $1970 \mathrm{~s},{ }^{11}$ with around 600000 annual procedures in the USA alone,${ }^{12}$ evidence of the effectiveness and cost-effectiveness of TKR in comparison to non-surgical treatments is warranted. ${ }^{7}$

In 2015, a randomised trial assessing the effectiveness of TKR plus non-surgical treatment as compared with nonsurgical treatment alone was published. ${ }^{13}$ Being the first of its kind, the study provided high-quality evidence on the effects of TKR and, at the same time, offered a unique opportunity to study the cost-effectiveness of TKR in two highly comparable treatment groups, thereby making an important contribution to previous non-randomised analyses of TKR cost-effectiveness. ${ }^{714}$

The purpose of the current study was to report the 24 months cost-effectiveness of TKR plus non-surgical treatment as compared with non-surgical treatment with the option of later TKR if needed using quality-adjusted life years (QALYs) data from the randomised trial and the unique Danish health registries which contain detailed information on health sector costs, social costs and prescription medication on the trial participants. We hypothesised that TKR plus non-surgical treatment would be a more cost-effective procedure compared with nonsurgical treatment with the option of later TKR if needed at 24 months due to greater improvements in quality of life counterbalancing the expected additional cost related to the surgery.

\section{METHODS}

\section{Study design}

This was a preplanned baseline to 24 months costutility analysis from a parallel group assessor-blinded randomised trial (1:1 ratio) that conforms to the Consolidated Health Economic Evaluation Reporting Standards statement for reporting health economic evaluations. ${ }^{15}$ Costs were collected from a health system perspective, with QALYs used as the outcome measure. Individual-level data were obtained from the clinical trial and linked with data from national registries for use in the analyses.

A brief presentation of the trial methods is provided below. Full details about the process for recruitment, criteria for eligibility, the randomisation procedure, allocation concealment and detailed description of the interventions have been published previously. ${ }^{16}$

\section{Participants}

One hundred patients diagnosed with symptomatic and moderate-to-severe radiographic knee OA considered eligible for TKR by the orthopaedic surgeon were included in the study. The study had three major exclusion criteria: (1) mean pain the previous week above $60 \mathrm{~mm}$ on a $100 \mathrm{~mm}$ Visual Analogue Scale, (2) previous knee replacement on the same side and (3) need for bilateral simultaneous TKR.

\section{Setting and time horizon}

Patients were recruited between September 2011 and December 2013 from one of two specialised, public outpatient clinics at Aalborg University Hospital, Denmark (Frederikshavn and Farsø), and all patients provided informed written consent before being enrolled. To have identical time periods for the whole population, we compared resource use and costs 1 year before randomisation (pre-period) to resource use and costs 2 years after randomisation for each individual patient.

\section{Randomisation procedure and allocation concealment}

The randomisation schedule was generated a priori in permuted blocks of eight, stratified by site and the allocation numbers were concealed in sealed, opaque envelopes prepared by an independent staff member. One research assistant at each of the two sites had access to the envelopes, opening them only after informed consent and baseline outcomes had been obtained.

\section{Comparators}

Patients were randomly assigned (1:1) to (1) undergo TKR plus 12 weeks of supervised non-surgical treatment or (2) receive only the 12 weeks of supervised non-surgical treatment.

\section{Total knee replacement}

A total cemented prosthesis with patellar resurfacing (NexGen, CR-Flex, fixed bearing or LPS-Flex, fixed bearing, Zimmer, Warsaw, Indiana, USA) was inserted by high-volume orthopaedic specialists (a surgeon performing +100 TKRs/year) using the surgical methods recommended by the manufacturer. ${ }^{17}$ Surgery was performed by the surgeon in charge of the assessment at the time of recruitment.

\section{Supervised non-surgical treatment}

The 12-week individualised, non-surgical treatment programme included exercise, patient education and insoles, with dietary advice and/or pain medication prescribed, if indicated. The treatments were delivered by physiotherapists and dietitians at Aalborg University Hospital, Denmark.

\section{Exercise}

The NEuroMuscular EXercise training programme (NEMEX), previously found feasible in patients with moderate-to-severe knee OA awaiting joint replacement, ${ }^{18}$ was administered in $60 \mathrm{~min}$ group-based sessions twice 
weekly supervised by a physiotherapist. To increase longterm adherence, after 12 weeks of exercise, the patients undertook a transition period of 8 weeks where the exercise programme was increasingly performed at home.

\section{Patient education}

Patient education was delivered as two 60 min group-based educational sessions which actively engaged the patients in their treatment. The sessions focused on disease characteristics, advice about treatment and self-help. Sessions were held in groups of up to 16 patients and were facilitated by the project physiotherapist.

\section{Dietary advice}

Patients with a body mass index $\geq 25$ at baseline had four individual 1-hour consultations with a dietitian with the overall aim of reducing body weight by at least $5 \% .{ }^{19}$ The programme was based on motivational interviewing. ${ }^{20}$

\section{Insoles}

Patients received individually fitted full-length Formthotics Original Dual Medium (perforated) insoles with medial arch support (Foot Science International, Christchurch, New Zealand). A $4^{\circ}$ lateral wedge was added to the insoles if patients had a knee-lateral-to-foot position (the knee moves over, or lateral, to the fifth toe in three or more of five trials). ${ }^{21}$

\section{Pain medication}

Paracetamol $1 \mathrm{~g}$ four times daily, ibuprofen $400 \mathrm{mg}$ three times daily and pantoprazole $20 \mathrm{mg}$ daily were prescribed by the orthopaedic surgeon if indicated. Prescriptions were reassessed every 3 weeks and the patients were instructed to contact the study team if they were uncertain about the need for continued pain medication.

\section{Booster sessions}

After the 12-week non-surgical programme and the 8-week transition period and until the 12-month follow-up, a physiotherapist phoned the patients monthly to support exercise adherence. Patients consulting the dietitian were telephoned twice by the dietitian to encourage dietary adherence.

\section{Patient and public involvement}

While no patients were involved in this cost-effectiveness analysis, the specific content of the non-surgical treatment was guided by feedback from patients to ensure feasibility and acceptance.

\section{Measurement of resource use and costs}

Information on resource use and costs, including healthcare costs and public transfer income for each patient, was retrieved from Danish national registries up until the 24-month follow-up. In Denmark, the Danish Civil Registration System assigns every citizen a personal identification number (central personal registration number), which allows for the linking of information between national registries at the individual level. This enables identification of the patients in the trial and calculations of costs associated with these individuals. Healthcare costs comprised expenses associated with inpatient services, outpatient visits, primary care services and prescription medication. Inpatient services were assessed as both including and excluding TKR surgeries during the study period. Data on inpatient and outpatient services are available from the National Patient Registry (NPR), which contains information on all kinds of patient contacts including diagnoses and diagnostic and treatment procedures. Linking data on resource use from NPR with the Danish Case Mix System (Diagnosis-Related Groups) enabled estimation of associated costs. Primary care included visits to the general practitioner, medical specialist, physiotherapist, chiropractor, laboratory work and others. Resources related to utilisation of the primary care services were derived from the Danish National Health Insurance Service Register. Costs were estimated for all prescription medication; pain medication (The Anatomical Therapeutic Chemical (ATC)-codes N02A, N02B and M01A) and non-pain medication (ie, anything else besides pain medication), respectively. Medication costs were calculated by multiplying the retail price with the prescribed quantity, available from the Danish Medicines Agency.

Non-protocol-driven resources, for example, costs of recruitment, were not included. As both groups received the same supervised non-surgical treatment (as described above), this cost was not included for either group. The cost of the non-surgical treatment was estimated to be between 560 Euros (actual cost of the non-surgical treatment in the trial) to 1646 Euros (estimated cost of the non-surgical treatment in private practice in Denmark) per person.

To increase the international applicability of the study, costs were adjusted to 2015-equivalent price levels using the consumer price index and converted to Euros (1 Euro=7.45 Danish Kroner); 1 Euro corresponded to 1.13 US dollars at the 2017 average exchange rate.

Public transfer income was calculated as the number of weeks a person was receiving sick leave pay, disability pension, early retirement and unemployment benefits (including activated persons). About half of the participants were older than 64 years $(56 \%)$, and retired (age pension). This information was available from national registries from Statistics Denmark.

\section{Measurement of effectiveness}

A generic measure of health in terms of QALYs gained was used as the effectiveness measure of effects up until the 24-month follow-up. This is a composite measure that considers both the quantity and quality of life of an individual. The maximum achievable health utility is 1 and hence, a QALY value of 1 reflects 1 year of full health, whereas a QALY value of 0 reflects death. Health-related quality of life (HRQoL; health utility) was measured using the three-level version of the EuroQol Group 5-Dimension Self-Report Questionnaire (EQ-5D), including the score 
on the descriptive index (ranging from -0.59 to 1.00 ) and the score on the Visual Analogue Scale (ranging from 0 to 100), ${ }^{22}$ at baseline, at 3 months, 6 months, 12 months and at the 24 months follow-up. The baseline to 12 months EQ-5D data were previously published in the primary randomisedcontrolled trial report, ${ }^{13}$ but has not previously been used for cost-effectiveness analyses. The EQ-5D-3L has five digits measuring mobility, self-care, usual activities, pain discomfort and anxiety/depression. The descriptive index is based on a Danish 'time trade-off' value set, ${ }^{23}$ which is a method used to evaluate the relative amount of time patients would be willing to sacrifice to avoid a certain poor health state. The patients completed the EQ-5D at baseline and all follow-up visits at the Department of Occupational Therapy and Physiotherapy, Aalborg University Hospital, Denmark.

\section{Analytical methods \\ Missing data}

Missing data were handled by using multiple imputation, which enables individuals with incomplete data to be included in the analysis. The underlying assumption when using multiple imputation is that data are missing at random, that is, the probability of missing values is not dependent on unobserved data. Missing data were imputed using multiple imputation in SAS, and the assumption of data missing at random was also tested and confirmed in SAS. Missing utility values occurred at 3, 6, 12 and 24 months, and thus, utilities were imputed at these time points using utilities from available time points.

\section{Costs in the pre-period, year 1 and year 2}

The costs of the two groups were compared by using arithmetic means for each period. The statistical significance of the difference between groups was assessed using the bootstrapped t-test.

\section{Cost-effectiveness analyses}

Regression analyses were used to estimate incremental costs and QALYs and data were analysed in accordance with intention-to-treat principle. Costs in the regression analyses only included healthcare costs. Because costs are normally right-skewed and QALYs left-skewed, a gamma distribution was assumed in the regression analyses. Both regression analyses were adjusted for covariates in the base-case analysis, that is, the cost regression was adjusted for age, sex and baseline costs and the QALY regression was adjusted for age, sex and baseline QALY. Two additional scenarios were also considered: one not taking covariates into account, that is, without adjustment (Scenario 1), and the other not considering either covariates or missing values/imputations (Scenario 2).

QALY gains or losses were calculated as the area under the curve, that is, taking changes in utility over time into account.

Costs and effects were discounted by $3 \%$.

\section{Subanalysis}

A subanalysis, including deaths during the study period, was conducted for each scenario (Base-case scenario, Scenario 1 and Scenario 2).

Sensitivity analyses

A probabilistic sensitivity analysis was carried out for each scenario in the primary analysis and the subanalysis, respectively. The probabilistic sensitivity analysis takes into account all parameter uncertainty at once. Incremental costs and QALYs were used to simulate 10000 random draws resulting in a scatterplot reflecting the probability of cost-effectiveness. In Denmark, no officially set willingness-to-pay threshold exists. Instead, we used a threshold of 22665 Euros/QALY or lower corresponding to the decision rule defined by the National Institute for Health and Care Excellence (NICE) ( 20000$).{ }^{24}$

A cost-effectiveness acceptability curve illustrating the cost-effectiveness at different thresholds and a costeffectiveness plane showing the uncertainty around the incrementalcost-effectiveness ratios (ICER) were produced (both excluding deaths).

All analyses were performed using SAS 9.1.3 (SAS Institute, North Carolina, USA) and the significance level was set to 0.05 .

\section{RESULTS}

\section{Patient characteristics}

The baseline characteristics of the two groups of patients and patient flow are presented in table 1 and figure 1 , respectively. Below $8 \%(n=117)$ of patients assessed for eligibility were excluded due to pain intensity above $60 \mathrm{~mm}$ out of $100 \mathrm{~mm}$.

Out of the 100 patients randomised, 24 months follow-up data were available for $47 / 50(94 \%)$ in the nonsurgical group and $43 / 50(86 \%)$ in the TKR plus nonsurgical group. Administrative data yielded that 16 out of 50 patients (32\%) from the non-surgical group had a TKR before the 24 months follow-up: 13 patients from baseline to 12 months and 3 patients between 12 and 24 months. Mean duration (range) from initiating the non-surgical treatment to the TKR was 8.7 (2.6 to 21.5) months. One of the 50 patients $(2 \%)$ in the TKR plus non-surgical group decided not to undergo TKR anyway. One patient in the TKR plus non-surgical group had three revision surgeries ending up with the prosthesis being removed and the knee fused following a deep infection. Due to severe knee stiffness during the rehabilitation period after TKR, three patients in the TKR plus non-surgical group and one patient in the non-surgical group who had TKR later required manipulation of the knee under anaesthesia. The mean follow-up time was 24.0 and 24.3 months in the TKR plus non-surgical group and the nonsurgical group, respectively.

Table 2 shows healthcare costs and public transfer income given as weeks in the pre-period, year 1 (12 months) and year 2 (24 months), respectively. The groups 
Table 1 Baseline characteristics in the randomised controlled trial of patients eligible for total knee replacement (TKR)

\begin{tabular}{|c|c|c|}
\hline Baseline characteristics & $\begin{array}{l}\text { TKR-+non-surgical group } \\
(\mathrm{n}=50)\end{array}$ & $\begin{array}{l}\text { Non-surgical group } \\
(\mathrm{n}=50)\end{array}$ \\
\hline Women, n (\%) & $32(64)$ & $30(60)$ \\
\hline Age (years), mean (SD) & $65.8(8.7)$ & $67.0(8.7)$ \\
\hline Body mass index, mean (SD) & $32.3(6.2)$ & $32.0(5.8)$ \\
\hline Bilateral knee pain, n (\%) & $18(36)$ & $17(34)$ \\
\hline \multicolumn{3}{|c|}{ Radiographic knee OA severity (Kellgren-Lawrence), n (\%) } \\
\hline Grade 1 & $0(0)$ & $0(0)$ \\
\hline Grade 2 & $7(14)$ & $5(10)$ \\
\hline Grade 3 & $21(42)$ & $21(42)$ \\
\hline Grade 4 & $22(44)$ & $24(48)$ \\
\hline \multicolumn{3}{|l|}{ KOOS } \\
\hline Pain & $48.6(17.5)$ & $49.5(13.1)$ \\
\hline Symptoms & $54.0(15.0)$ & $58.3(15.2)$ \\
\hline ADL & $55.0(17.0)$ & $53.5(14.2)$ \\
\hline Sport/rec & $18.0(14.7)$ & $16.7(15.1)$ \\
\hline QOL & $32.3(15.3)$ & $32.7(13.3)$ \\
\hline $\mathrm{KOOS}_{4}$ & $47.4(13.4)$ & $48.5(11.4)$ \\
\hline Timed Up and Go test, seconds & $9.4(2.4)$ & $8.6(2.1)$ \\
\hline 20-metre walk test, seconds & $13.4(3.7)$ & $12.2(2.6)$ \\
\hline Used pain medication in the last week, yes $n(\%)$ & $33(67)$ & $29(58)$ \\
\hline
\end{tabular}

Radiographic severity: Radiographic knee osteoarthritis severity on the Kellgren-Lawrence scale; KOOS : The mean score of four out of five of the Knee injury and Osteoarthritis Outcome Score subscales covering Pain, Symptoms, Function in daily living (ADL), Sport/Rec: Function in sport and recreation. and Quality of life (QOL), with scores ranging from 0 to 100 (worst to best scale). OA, osteoarthritis.

had similar healthcare costs during the year prior to randomisation (2695 vs 2644 Euros). At 12 months after randomisation, healthcare costs in the TKR plus nonsurgical group were more than double those of the nonsurgical group (16343 vs 7028 Euros), mostly due to the surgical procedure. Although not statistically significant, the costs in the TKR plus non-surgical group were lower at the 24 months follow-up (6733 vs 7486 Euros) because some patients in the non-surgical group underwent TKR. No significant between-group differences were found in weeks of incurring public transfer income.

The non-surgical group experienced a gain in health utilities of 0.056 from baseline to 24 months while the TKR plus non-surgical group experienced a gain of 0.195 , with the largest increases in health utilities in both groups from baseline to 3 months (see table 3 for utility values at the different time points).

Incremental costs and QALYs for each scenario are presented in table 4 . In all scenarios, TKR plus nonsurgical treatment was more expensive, but also more effective in terms of QALY gain. ICERs and the probability of cost-effectiveness at the willingness-to-pay threshold for each scenario are also presented in table 4. In the Basecase (adjusted) scenario, TKR plus non-surgical treatment costed 32611 Euros per QALY gained, which is above the threshold for willingness-to-pay defined by NICE (22665
Euros/QALY). However, in the unadjusted Scenario 1 and unadjusted and without imputation of missing values (Scenario 2) the ICERs were below the threshold (19917 Euros/QALY and 18497 Euros/QALY, respectively). The probability of cost-effectiveness of TKR plus non-surgical treatment was only $23.2 \%$ in the (adjusted) Base-case scenario, but increased to $58.3 \%$ and $61.9 \%$ in Scenarios 1 and 2, respectively.

Cost-effectiveness acceptability curve showing the probability of TKR plus non-surgical treatment being costeffective at different thresholds is presented in figure 2. The probability of cost-effectiveness was below $60 \%$ up until a threshold of approximately 40000 Euros/QALY. To reach a probability of cost-effectiveness greater than $90 \%$, a threshold of minimum 60000 Euros/QALY was needed.

Cost-effectiveness plane illustrating the uncertainty around the ICER is presented in online supplementary appendix figure 1 .

\section{Subanalysis including deaths}

Three persons died in the TKR plus non-surgical treatment group and one person in the non-surgical treatment only group. Including deaths in the analysis decreased the QALY gained in both groups. The non-surgical group experienced a gain in health utilities of 0.040 from 


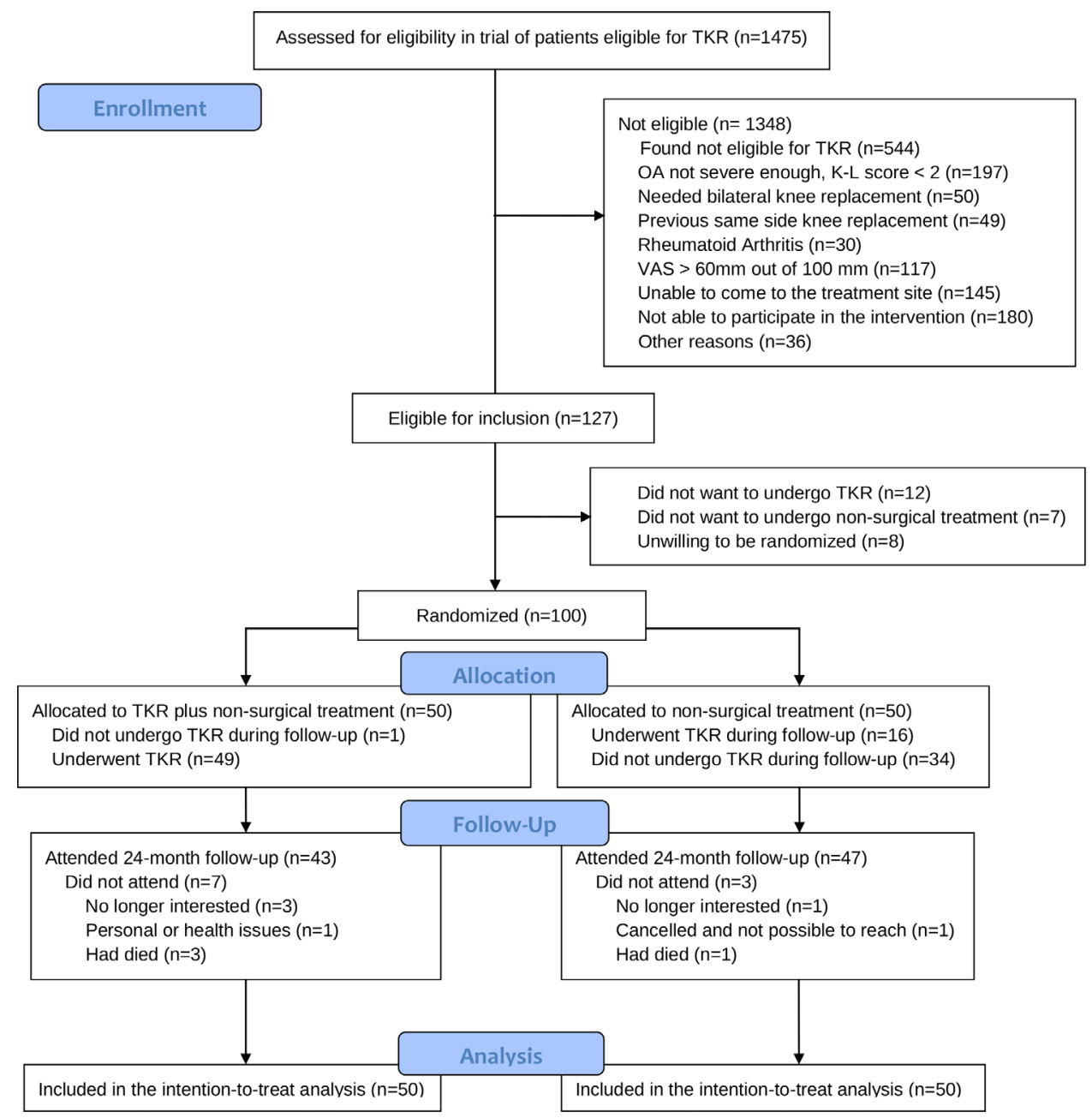

Figure 1 Flow of patients in the randomised controlled trial of patients eligible for total knee replacement. K-L score, KellgrenLawrence score; OA,osteoarthritis; TKR,total knee replacement; VAS, Visual Analogue Scale.

baseline to 24 months while the TKR plus non-surgical group experienced a gain of 0.136 , with the largest increases in health utilities in both groups from baseline to 3 months (see online supplementary appendix table 1 for utility values at the different time points).

Including deaths in the regression analysis changed the estimates of incremental costs and QALYs (online supplementary appendix table 2). TKR plus non-surgical treatment was still more expensive and more effective for all scenarios but in all three scenarios the ICER exceeded the NICE threshold. In the Base-case scenario, the ICER was more than twice as high as the threshold for willingnessto-pay defined by NICE (22665 Euros/QALY), and the probability of cost-effectiveness was only $7.8 \%$. In Scenarios 1 and 2 the probability of cost-effectiveness was $12.4 \%$ and $13.8 \%$, respectively.

\section{DISCUSSION}

TKR plus non-surgical treatment appear to be more expensive, but also more effective than non-surgical treatment after 24 months in patients with knee OA eligible for TKR and moderate intensity pain. The cost-utility analysis suggested that TKR plus non-surgical treatment was not cost-effective compared with non-surgical treatment with the option of later TKR if needed from a 24-month health system perspective in secondary care in Denmark when adjusting for covariates and imputing missing values. Results were sensitive to changes, as the treatment was cost-effective in the unadjusted scenario, highlighting the need for further research with 5 to 10-year time horizons.

Given the extensive burden of knee $\mathrm{OA}^{3}{ }^{34}$ there is considerable societal demand for evidence on costeffective evidence-based treatments. ${ }^{25}$ The current study provides the first direct comparison of two different treatment strategies in terms of cost-effectiveness after 24 months for patients with moderate-to-severe symptomatic and radiographic knee OA. The cost-utility analysis was conducted alongside a randomised trial, which demonstrated that TKR plus non-surgical treatment compared with non-surgical treatment was twice as effective in terms of pain relief and functional improvements after 12 and 24 months. ${ }^{1326}$ Therefore, we hypothesised that TKR would be a cost-effective procedure after 24 months due to higher improvements in quality of life counterbalancing the expected additional cost related to the procedure. However, in contrast to our hypothesis, TKR plus 


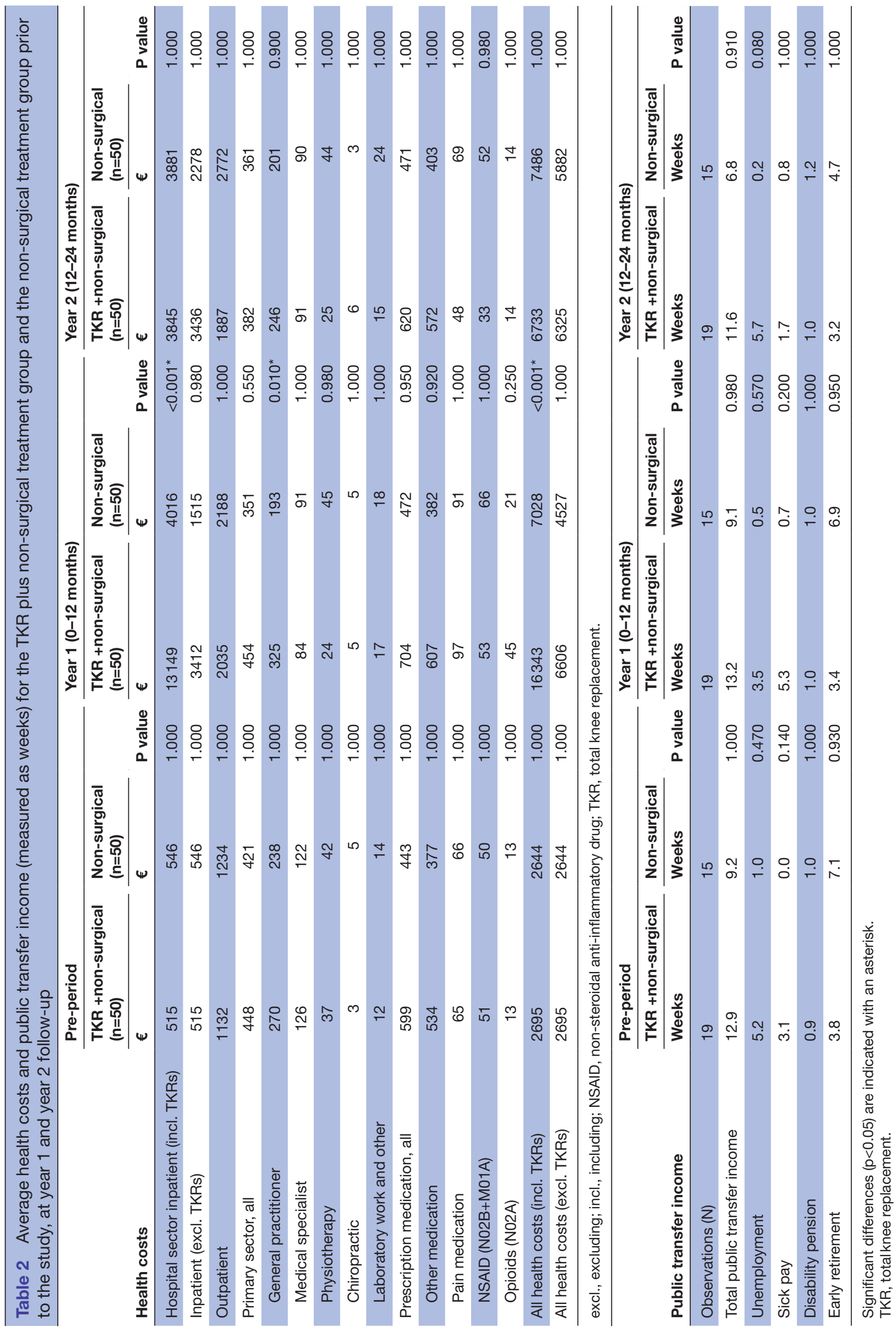




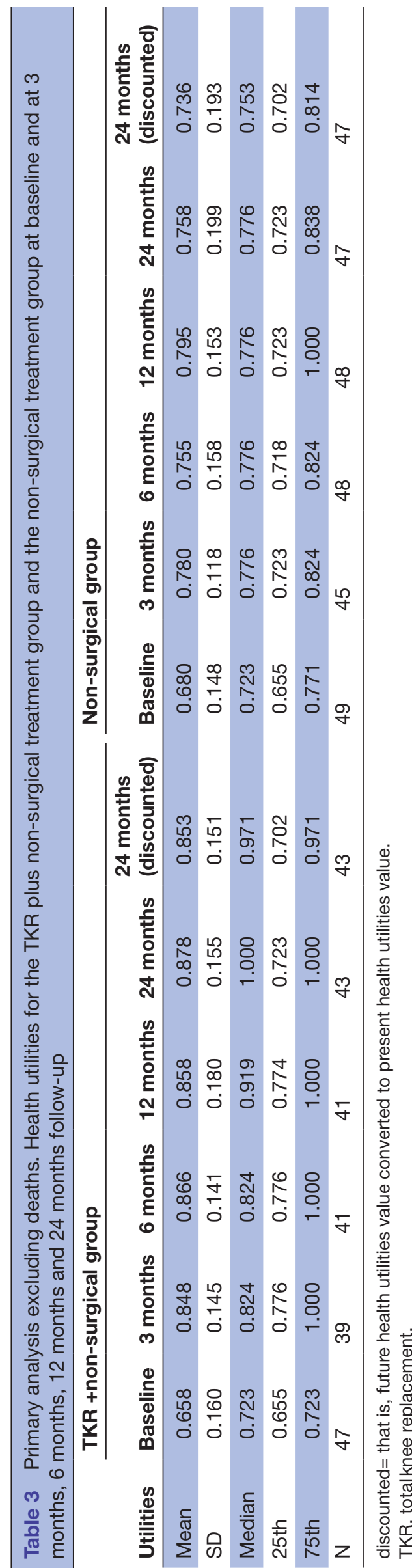

non-surgical treatment was not found to be cost-effective compared with non-surgical treatment with the option of later TKR if needed from a 24 months perspective in secondary care in Denmark. The cost per QALY gained exceeded the threshold defined by NICE by approximately 10000 Euros. ${ }^{24}$ However, without adjustment for covariates and imputation of missing values the cost per QALY was just cost-effective according to the threshold (ICER of 18497 Euros/QALY).

Our results from the Base-case scenario contrast with findings in a recent systematic review. ${ }^{27}$ The review included four studies examining the cost-effectiveness of TKR compared with non-surgical procedures and all four concluded that TKR was a cost-effective option. However, as opposed to our study, none of the previous studies were based on a randomised trial and the content of the non-surgical treatment was neither as comprehensive nor aligned with evidence-based recommendations as the non-surgical treatment in our study. Two of the previous studies used a Markov model to assess the long-term and lifetime cost-effectiveness of TKR. ${ }^{28}{ }^{29}$ The remaining two were cohort-based studies examining shortterm cost-effectiveness of TKR..$^{30}{ }^{31}$ A recent cohort-based cost-effectiveness analysis, not included in the systematic review, concluded that TKR was not cost-effective at a group level over 8 years, while it would be cost-effective if it was restricted to patients with more severe symptoms. ${ }^{14}$ In contrast, we did not find that TKR was cost-effective in addition to non-surgical treatment after 24 months in patients with moderate intensity pain. Our study provides the first cost-effectiveness analysis of TKR in addition to recommended non-surgical treatment using two comparable treatment groups, thereby providing an important addition to the above-mentioned non-randomised studies.

One could argue that extending the time horizon might have led to a different conclusion. If the positive effect of the surgery persists beyond the 24 months, TKR plus non-surgical treatment might eventually end up being a cost-effective option. Though the mean utility fluctuates slightly over time in both groups, there seems to be an overall improvement in the TKR plus non-surgical group as compared with non-surgical treatment only. Assuming that this between-group difference is at least maintained and a potential increased cost in the non-surgical group due to future TKR surgery, this could improve the costeffectiveness ratios in favour of TKR plus non-surgical treatment. However, as indicated by a previous report, ${ }^{32}$ improvements in symptoms might decline from 1 to 5 years after TKR, questioning the assumptions underlining a potential long-term cost-effectiveness of TKR. In the TKR plus non-surgical group, three people died during the period, while only one person died in the nonsurgical group. When including the deaths in the analysis, TKR plus non-surgical treatment was still more effective than non-surgical treatment, though not as effective as in the primary analysis. This is because death corresponds to a QALY value of 0 , thereby attenuating the effect of the surgery. 
Table 4 Primary analysis excluding deaths. Incremental cost-effectiveness ratios (ICERs) and probability of cost-effectiveness of TKR plus non-surgical treatment versus non-surgical treatment alone for each scenario

\begin{tabular}{|c|c|c|c|c|c|c|}
\hline \multirow[b]{2}{*}{ Analysis } & \multirow{2}{*}{ 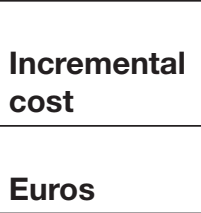 } & \multirow[b]{2}{*}{$95 \% \mathrm{Cl}$} & \multirow{2}{*}{ 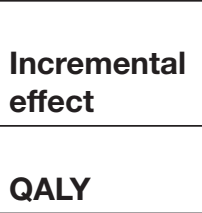 } & \multirow[b]{2}{*}{$95 \% \mathrm{Cl}$} & \multirow{2}{*}{$\begin{array}{l}\text { ICER } \\
\text { Euros/ } \\
\text { QALY }\end{array}$} & \multirow{2}{*}{$\begin{array}{l}\text { Probability of cost- } \\
\text { effectiveness at } 22665 \\
\text { Euros } \\
\% \\
\%\end{array}$} \\
\hline & & & & & & \\
\hline Base-case & 6070 & 1857 to 10283 & 0.186 & 0.078 to 0.294 & 32611 & 23.2 \\
\hline Scenario 1 & 4640 & -200 to 9480 & 0.233 & 0.088 to 0.378 & 19917 & 58.3 \\
\hline
\end{tabular}

Base-case=adjusted for age, sex and baseline value; Scenario 1=unadjusted; Scenario 2=unadjusted and without imputation of missing values.

QALY, quality-adjusted life year; TKR, total knee replacement.

\section{Strengths and limitations}

All treatments, in particular surgical treatment, are associated with placebo effects. ${ }^{33}$ As our study did not include a sham surgery control group, we were not able to evaluate the proportion of the 24 months treatment effects attributable to contextual factors. ${ }^{34}$ Neither did we include a group receiving TKR without the non-surgical treatment, leaving us without the possibility of evaluating the additional effect and cost of the non-surgical treatment. As 32\% from the non-surgical group had TKR surgery during the 24 months, it is likely that the true additional effect and cost of TKR have been underestimated in the study. Furthermore, as one of the exclusion criteria was mean pain the previous week above $60 \mathrm{~mm}$ on a $100 \mathrm{~mm}$ Visual Analogue Scale, our results might not be generalisable to patients with more severe pain at baseline. However, $42 \%$ of the patients reported pain higher than $60 \mathrm{~mm}$ when asked about worst pain during the previous 24 hours and the mean pain intensity in our trial of 49 on a 0 to 100 worst to best scale is comparable to a range of previous clinical studies evaluating pain severity prior to TKR. ${ }^{35-37}$ Additionally, the effects from non-surgical treatments, such as exercise, does not seem to be associated with pain severity at baseline, ${ }^{38}$ suggesting that the nonsurgical treatment might be as effective in patients with more severe pain. The short time horizon and the different findings in the analysis without adjustment for covariates and imputation of missing values and the subanalysis including deaths emphasise the susceptibility of the results and highlight the need for further analyses in the field including follow-ups at 5 to 10 years. The study strengths include the highly comparable treatment groups as a result of the randomisation and the use of data from the unique Danish registries, which comprise data deemed to be of high quality. Linkage between these registries and the Danish Civil Registration system allowed for retrieving data on an individual level, which is a unique feature of this study.

\section{CONCLUSIONS}

From a 24 months perspective in secondary care in Denmark, TKR plus non-surgical treatment does not appear to be cost-effective compared with non-surgical treatment with the option of later TKR if needed in patients with moderate-to-severe osteoarthritis and

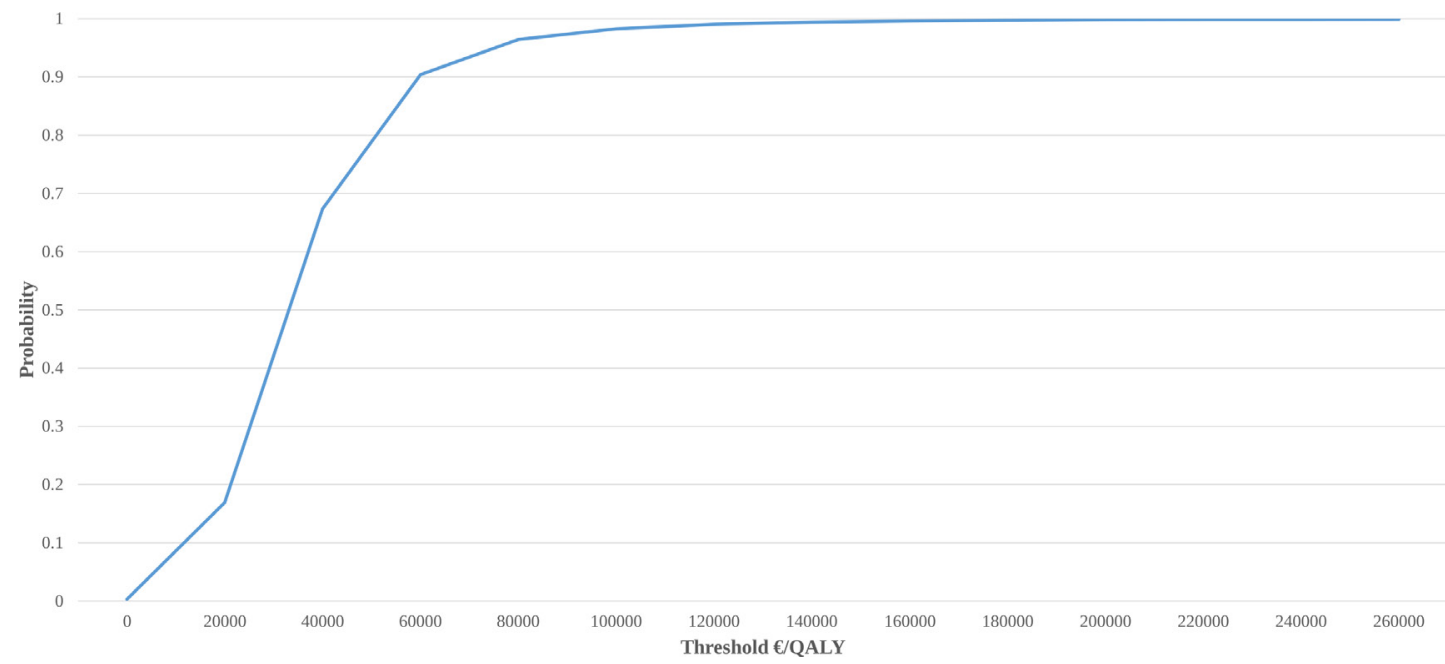

Figure 2 Cost-effectiveness acceptability curve illustrating the probability of TKR plus non-surgical treatment being costeffective at different thresholds (excluding deaths). QALY,quality-adjusted life years; TKR, total knee replacement. 
moderate intensity pain, eligible for TKR. However, as TKR plus non-surgical treatment was just cost-effective when not adjusting for covariates and not imputing missing values, further confirmatory studies with longer follow-up are needed.

\section{Author affiliations}

${ }^{1}$ Research Unit for Musculoskeletal Function and Physiotherapy, Department of Sports Science and Clinical Biomechanics, Syddansk Universitet, Odense, Syddanmark, Denmark

2Department of Physiotherapy and Occupational Therapy, Næstved-SlagelseRingsted Hospitals, Region Zealand, Slagelse, Denmark

${ }^{3}$ Orthopedic Surgery Research Unit, Aalborg University Hospital, Aalborg, Denmark ${ }^{4}$ Center for Sensory-Motor Interaction (SMI), Department of Health Science and Technology, Aalborg University, Aalborg, Denmark

${ }^{5}$ Department of Clinical Medicine, Aalborg Universitet, Aalborg, Denmark

${ }^{6} / 2$ minds, Aarhus, Denmark

${ }^{7}$ VIVE - The Danish Center for Social Science Research, Copenhagen, Hovedstaden, Denmark

\section{Twitter Søren Thorgaard Skou @STSkou and Ewa Roos @ewa_roos}

Acknowledgements We thank the orthopaedic surgeons and other healthcare personnel from the Department of Orthopedic Surgery, Aalborg University Hospital, for their involvement in the recruitment of patients for the two studies; the Department of Occupational Therapy and Physiotherapy, Aalborg University Hospital, Denmark, for allowing us to use their facilities for the treatment and outcome assessments; project workers Anders Bundgaard Lind, Anders Norge Jensen, Anna Emilie Livbjerg, Dorte Rasmussen, Helle Mohr Brøcher, Henriette Duve, Janus Duus Christiansen, Josephine Nielsen, Kate Mcgirr, Lasse Lengsø, Lonneke Hjermitslev, Malene Daugaard, Maria Helena Odefey, Mette Bøgedal, Mikkel Simonsen, Niels Balslev, Rikke Elholm Jensen and Svend Lyhne for helping with administrative tasks, data collection, data entry and treatment. Finally, the study funders and patients participating should be acknowledged because without their participation it would not have been possible to conduct the trials.

Contributors Study conception and design: STS, JK, ER, ML, LA-N, SR, OS. Recruitment of patients: ML, OS. Acquisition of data: STS, JK. Analysis and interpretation of data: STS, JK, ER, ML, LA-N, RI, ATL, SR, OS. Drafting the article or revising it critically for important intellectual content: STS, JK, ER, ML, LA-N, RI, ATL, SR, OS. Final approval of the article: STS, JK, ER, ML, LA-N, RI, ATL, SR, OS. All authors had full access to all the data (including statistical reports and tables) in the study and take responsibility for the integrity of the data and the accuracy of the data analysis.

Funding The work was supported by The Danish Rheumatism Association, The Health Science Foundation of North Denmark Region, Obel Family Foundation, Foot Science International, Spar Nord Foundation, The Bevica Foundation, The Association of Danish Physiotherapists Research Fund, Medical Specialist Heinrich Kopp's Grant and The Danish Medical Association Research Fund. Dr Skou is currently funded by a grant from Region Zealand and a grant the European Research Council (ERC) under the European Union's Horizon 2020 research and innovation programme (grant agreement № 801790).

Disclaimer The funders played no role in the design and conduct of the study; collection, management, analysis and interpretation of the data and preparation, review or approval of the manuscript or decision to submit the manuscript for publication.

Competing interests None declared.

Patient consent for publication Not required.

Ethics approval The study was designed to follow the principles of the Declaration of Helsinki and ethics approval was obtained from the local Ethics Committee of The North Denmark Region (N-20110024).

Provenance and peer review Not commissioned; externally peer reviewed.

Data availability statement Data may be obtained from a third party and are not publicly available. The data that support the findings of this study are available from Statistics Denmark, but restrictions apply to the availability of these data, which were used under license for the current study, and so are not publicly available. Data are however available from the authors $\mathrm{OS}$ and $\mathrm{ML}$ upon reasonable request and with permission of Statistics Denmark.
Open access This is an open access article distributed in accordance with the Creative Commons Attribution Non Commercial (CC BY-NC 4.0) license, which permits others to distribute, remix, adapt, build upon this work non-commercially, and license their derivative works on different terms, provided the original work is properly cited, appropriate credit is given, any changes made indicated, and the use is non-commercial. See: http://creativecommons.org/licenses/by-nc/4.0/.

ORCID iDs

Søren Thorgaard Skou http://orcid.org/0000-0003-4336-7059

Ewa Roos http://orcid.org/0000-0001-5425-2199

\section{REFERENCES}

1 Cross M, Smith E, Hoy D, et al. The global burden of hip and knee osteoarthritis: estimates from the global burden of disease 2010 study. Ann Rheum Dis 2014;73:1323-30.

2 Peat G, McCarney R, Croft P. Knee pain and osteoarthritis in older adults: a review of community burden and current use of primary health care. Ann Rheum Dis 2001;60:91-7.

3 Vos T, Flaxman AD, Naghavi M, et al. Years lived with disability (YLDs) for 1160 sequelae of 289 diseases and injuries 1990-2010: a systematic analysis for the global burden of disease study 2010. Lancet 2012;380:2163-96.

4 Salmon JH, Rat AC, Sellam J, et al. Economic impact of lower-limb osteoarthritis worldwide: a systematic review of cost-of-illness studies. Osteoarthritis Cartilage 2016;24:1500-8.

5 Nguyen U-SDT, Zhang Y, Zhu Y, et al. Increasing prevalence of knee pain and symptomatic knee osteoarthritis: survey and cohort data. Ann Intern Med 2011;155:725-32.

6 Carr AJ, Robertsson O, Graves S, et al. Knee replacement. Lancet 2012;379:1331-40.

7 Nwachukwu BU, Bozic KJ, Schairer WW, et al. Current status of cost utility analyses in total joint arthroplasty: a systematic review. Clin Orthop Relat Res 2015;473:1815-27.

8 Beswick AD, Wylde V, Gooberman-Hill R, et al. What proportion of patients report long-term pain after total hip or knee replacement for osteoarthritis? A systematic review of prospective studies in unselected patients. BMJ Open 2012;2:e000435.

9 Liddle AD, Judge A, Pandit $\mathrm{H}$, et al. Adverse outcomes after total and unicompartmental knee replacement in 101,330 matched patients: a study of data from the National joint Registry for England and Wales. Lancet 2014;384:1437-45.

10 Nelson AE, Allen KD, Golightly YM, et al. A systematic review of recommendations and guidelines for the management of osteoarthritis: the chronic osteoarthritis management initiative of the U.S. bone and joint initiative. Semin Arthritis Rheum 2014;43:701-12.

11 Singh JA, Vessely MB, Harmsen WS, et al. A population-based study of trends in the use of total hip and total knee arthroplasty, 19692008. Mayo Clin Proc 2010;85:898-904.

12 Agency for Healthcare Research and Quality. National statistics on 152 arthroplasty knee, HCUPnet, healthcare cost and utilization project, 2013. Available: http://hcupnet.ahrq.gov/

13 Skou ST, Roos EM, Laursen MB, et al. A randomized, controlled trial of total knee replacement. N Engl J Med 2015;373:1597-606.

14 Ferket BS, Feldman Z, Zhou J, et al. Impact of total knee replacement practice: cost effectiveness analysis of data from the osteoarthritis initiative. BMJ 2017;356:j1131.

15 Husereau D, Drummond M, Petrou S, et al. Consolidated health economic evaluation reporting standards (cheers) statement. BMJ 2013;346:f1049.

16 Skou ST, Roos EM, Laursen MB, et al. Total knee replacement plus physical and medical therapy or treatment with physical and medical therapy alone: a randomised controlled trial in patients with knee osteoarthritis (the MEDIC-study). BMC Musculoskelet Disord 2012;13:67.

17 Endres S. High-flexion versus conventional total knee arthroplasty: a 5-year study. J Orthop Surg 2011;19:226-9.

18 Ageberg E, Link A, Roos EM. Feasibility of neuromuscular training in patients with severe hip or knee oa: the individualized goalbased NEMEX-TJR training program. BMC Musculoskelet Disord 2010;11:126.

19 Christensen R, Bartels EM, Astrup A, et al. Effect of weight reduction in obese patients diagnosed with knee osteoarthritis: a systematic review and meta-analysis. Ann Rheum Dis 2007;66:433-9.

20 Miller WR, Rollnick S. Motivational interviewing: preparing people for change. New York: Guilford Press, 2002.

21 Ageberg E, Bennell KL, Hunt MA, et al. Validity and inter-rater reliability of medio-lateral knee motion observed during a single-limb mini squat. BMC Musculoskelet Disord 2010;11:265. 
22 Szende A, Williams A. Measuring self-reported population health: an international perspective based on EQ-5D. Budapest: SpringMed Publishing, 2004

23 Wittrup-Jensen KU, Lauridsen J, Gudex C, et al. Generation of a Danish TTO value set for EQ-5D health states. Scand J Public Health 2009;37:459-66.

24 National Institute for Health Care Excellence. Guide to the methods of technology appraisal 2013. London: National Institute for Health and Care Excellence (NICE), 2013.

25 Losina E, Paltiel AD, Weinstein AM, et al. Lifetime medical costs of knee osteoarthritis management in the United States: impact of extending indications for total knee arthroplasty. Arthritis Care Res (Hoboken) 2015;67:203-15.

26 Skou ST, Roos EM, Laursen MB, et al. Total knee replacement and non-surgical treatment of knee osteoarthritis: 2-year outcome from two parallel randomized controlled trials. Osteoarthritis Cartilage 2018;26:1170-80.

27 Kamaruzaman H, Kinghorn P, Oppong R. Cost-Effectiveness of surgical interventions for the management of osteoarthritis: a systematic review of the literature. BMC Musculoskelet Disord 2017;18:183.

28 Ruiz D, Koenig L, Dall TM, et al. The direct and indirect costs to Society of treatment for end-stage knee osteoarthritis. J Bone Joint Surg Am 2013;95:1473-80.

29 Bedair H, Cha TD, Hansen VJ. Economic benefit to Society at large of total knee arthroplasty in younger patients: a Markov analysis. $J$ Bone Joint Surg Am 2014;96:119-26.

30 Waimann CA, Fernandez-Mazarambroz RJ, Cantor SB, et al. Costeffectiveness of total knee replacement: a prospective cohort study. Arthritis Care Res (Hoboken) 2014;66:592-9.
31 Räsänen P, Paavolainen P, Sintonen H, et al. Effectiveness of hip or knee replacement surgery in terms of quality-adjusted life years and costs. Acta Orthop 2007;78:108-15.

32 Nilsdotter A-K, Toksvig-Larsen S, Roos EM. A 5 year prospective study of patient-relevant outcomes after total knee replacement. Osteoarthritis Cartilage 2009;17:601-6.

33 Wartolowska K, Judge A, Hopewell S, et al. Use of placebo controls in the evaluation of surgery: systematic review. BMJ 2014;348:g3253

34 Zou K, Wong J, Abdullah N, et al. Examination of overall treatment effect and the proportion attributable to contextual effect in osteoarthritis: meta-analysis of randomised controlled trials. Ann Rheum Dis 2016;75:1964-70.

35 Gossec L, Paternotte S, Maillefert JF, et al. The role of pain and functional impairment in the decision to recommend total joint replacement in hip and knee osteoarthritis: an international crosssectional study of 1909 patients. Report of the OARSI-OMERACT Task force on total joint replacement. Osteoarthritis Cartilage 2011;19:147-54.

36 Keurentjes JC, Fiocco M, So-Osman C, et al. Patients with severe radiographic osteoarthritis have a better prognosis in physical functioning after hip and knee replacement: a cohort-study. PLoS One 2013;8:e59500.

37 Wise BL, Niu J, Felson DT, et al. Functional impairment is a risk factor for knee replacement in the multicenter osteoarthritis study. Clin Orthop Relat Res 2015;473:2505-13.

38 Juhl C, Christensen R, Roos EM, et al. Impact of exercise type and dose on pain and disability in knee osteoarthritis: a systematic review and meta-regression analysis of randomized controlled trials. Arthritis Rheumatol 2014;66:622-36. 